\title{
Recruiting Primary Care Practices for Research: Reflections and Reminders
}

\author{
Douglas H. Fernald, MA, Bonnie T. Jortberg, PhD, RD, CDE, \\ Danielle M. Hessler, PhD, Robyn Wearner, RD, MA, L. Miriam Dickinson, PhD, \\ Lawrence Fisher, PhD, and W. Perry Dickinson, $M D$
}

Background: Recruiting primary care practices for research projects has always required carefully tended relationships, a compelling message, and good timing. Recruiting practices to participate in practice transformation research trials may take more and different efforts. We reflect on practice recruitment for a recently-concluded trial of a diabetes self-management support system in 2 states and 36 practices.

Methods: Iterative qualitative analysis of field notes, semistructured clinician and staff interviews, and meeting notes from a 2-state, cluster-randomized trial that aimed to improve self-management support for patients with type 2 diabetes mellitus.

Results: Although all 36 enrolled practices finished the study, fully recruiting practices early on took considerable effort, yielding 2 primary lessons: 1) practice-based research networks (PBRNs) must recruit more stakeholders at more levels, at the clinic, in the system, and across roles; and 2) practice recruitment is a process and may take longer than expected with unplanned turnover of key contacts. Adjusting our recruitment strategies required: helping with communication efforts in practices; aligning our study message according to stakeholders' interests; allowing for minor adaptations at the practicelevel to align with critical practice workflows, staffing, and resources; re-engaging with clinical leadership over time; and identifying a "backup" champion due to turnover.

Conclusions: When undertaking a pragmatic clinical trial requiring substantial practice change in a PBRN setting across a large number of practices, it is important that PBRN leaders develop a comprehensive strategy to identify and engage a broad group of stakeholders within each practice, understand their needs and priorities around research, and design and implement a structured communications strategy to maintain engagement throughout every phase of the project. (J Am Board Fam Med 2018;31:947-951.)

Keywords: Ambulatory Care Facilities, Health Services Research, Leadership, Primary Health Care, Self-Management, Type 2 Diabetes Mellitus, Workflow

Recruiting primary care practices for research has always required carefully tended relationships, a compelling message, and good timing. As primary care is beset with a panoply of ever-evolving quality

This article was externally peer reviewed.

Submitted 20 January 2018; revised 7 May 2018; accepted 25 May 2018.

From the Department of Family Medicine, University of Colorado School of Medicine, Aurora, CO (DHF, BTJ, RW, LMD, WPD); Department of Family and Community Medicine, University of California-San Francisco, San Francisco, CA (DMH, LF).

Funding: National Institute of Diabetes and Digestive and Kidney Diseases 1R18DK096387-01.

Conflict of interest: none declared.

Corresponding author: Douglas H. Fernald, MA, University of Colorado School of Medicine, Department of Family initiatives, multiple pressures for transformation, and slimmer margins, recruiting practices for research trials may take more and different efforts and extra attention. This may be especially true for projects that require extensive redesign and implementation work-basically requiring real change on the part of the practices. Previous studies have reported on clinician and practice recruitment strategies that include using a personal approach, getting staff buy-in, streamlining the research process, reducing clinician burden of research activi-

Medicine, 12631 E. 17th Ave., Mail Stop F496 Aurora, CO 80045-0508 (E-mail: doug.fernald@ucdenver.edu). 
ties, training staff on research activities, and using a tailored, iterative approach. ${ }^{1-8}$

Improving the quality of care for patients with type 2 diabetes mellitus (T2DM) remains a consistent priority in research and in quality programs for primary care. Self-management support (SMS) for patients is among the components of national quality programs. ${ }^{9,10}$ SMS requires functional teambased care across well-coordinated staff and clinician roles. Yet, few tools are available to assist practices and patients with SMS for diabetes. The University of Colorado Denver and University of California San Francisco tested a comprehensive, evidence-based SMS program that supports behavior change through an interactive behavior change technology. The 3-arm, cluster-randomized trial evaluated the implementation of the Connection to Health (CTH) SMS for T2DM program in primary care practices. Many of our earlier practicebased research projects have been limited, focused, short-term research efforts that could be accomplished by a subset of the practice without changing roles and workflow. However, fully implementing SMS, including multiple components (such as registries, action plans, patient assessment and followup) may require substantial redesign and engagement of more roles beyond the clinician. ${ }^{11}$ The current literature does not fully address best practices around whole-practice recruitment for practice transformation research trials that require significant practice-level change around workflow or staffing. We conducted a qualitative analysis of unstructured and semistructured data collected during the recruitment process for a recently completed trial to identify specific strategies to identify recruitment strategies that may be helpful for similar studies in the future.

\section{Methods}

The Connection to Health study aimed to: 1) examine the reach, effectiveness, adoption, implementation, and maintenance (RE-AIM) ${ }^{12,13}$ of CTH for patients with T2DM; 2) determine the incremental benefit of brief targeted practice facilitation on the implementation of $\mathrm{CTH}$; and 3) identify practice characteristics that affect CTH RE-AIM outcomes. Practices were randomly assigned to receive and implement the CTH program, the CTH program plus practice facilitation, or an SMS academic detailing educational inter- vention. ${ }^{14}$ The study design called for 18 family medicine or internal medicine practices each in Colorado and California, with equal numbers randomized to each of the 3 arms. From the project start date, the study design planned for a total recruitment window of 27 months, recruiting practices in 3 waves in both states. The intervention phase for the first wave of practices was planned to start at 15 months from the project start date.

Along with the overall recruitment plan, the study team prepared several documents to introduce the study, including a 3-page study brief and a letter of agreement listing participation requirements for a practice. A general study announcement was also published in Colorado's practicebased research network (PBRN) newsletter and the state chapter of the family physician professional society. Practices were recruited continuously from the beginning of the study until the cohort was completed (June 2012 through October 2015).

For this analysis on recruitment, we used a subset of study data: all facilitator field notes through the first SMS practice training, baseline clinician and staff semistructured interviews notes and transcripts, and meeting notes specifically addressing recruitment. Field notes and interview data were available for all 36 practices which ultimately enrolled in the study. Only meeting notes were available for data on practices that did not enroll.

A team-based, iterative analysis began with an initial "immersion/crystallization" review of the data. ${ }^{15}$ Immersion/crystallization is an analytic style that involves cycles of concentrated reading and review of the text data, combined with reflection and intuitive insights, until reportable interpretation becomes apparent. ${ }^{16}$ From the immersion/crystallization phase, an initial set of high-level conceptual categories was developed for template coding to segment all the available field note and interview data. ${ }^{17} \mathrm{~A}$ case-based matrix was used to organize emerging findings about practice recruitment. ${ }^{18} \mathrm{~A}$ final set of findings was reviewed and refined by the authors. This study was reviewed and approved by the Colorado Multiple Institutional Review Board and the University of California San Francisco Institutional Review Board.

\section{Results}

More than 20 practices or practice systems were contacted to participate in the project, which in- 
Figure 1. Planned and actual recruitment and intervention timeline.

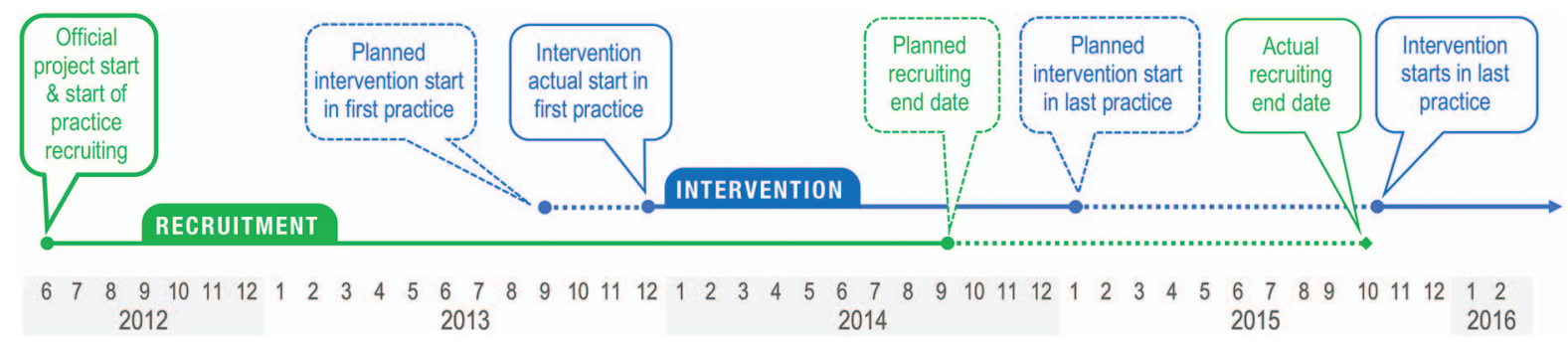

cluded meeting with clinical directors and practice stakeholders to provide them with sufficient information for them to make an informed decision about whether to participate. Thirty-six primary care practices were successfully enrolled in the study. Most practices were family medicine (23/36, $63.9 \%)$, were urban/suburban $(26 / 36,72.2 \%)$, and had an average of almost 7 (6.89) clinicians. Recruitment was more complex and took longer than anticipated, even for our team that has extensive experience in PBRNs. Twenty-seven months were planned for recruiting all 36 practices; however, the final recruitment period required an additional 13 months (Figure 1).

Compared with prior, more limited studies, this project involving the implementation of new processes for SMS and team-based care (and in 2 arms, a health information technology solution) required engagement and acceptance of many more levels within practices or systems, and a longer recruitment process. Finding alignment with practice and system interests was essential. Among the practices that participated, system- or practice-level leadership expressed that the study aligned with interests to improve the care of T2DM patients, primarily by offering additional tools and resources to clinicians and staff to enhance care delivery or to meet external requirements. As 1 system leader in California described it, the study was an opportunity to "reboot" their SMS efforts and to fulfill required quality reporting toward eventual reimbursement for SMS activities. Importantly, system leaders and practice leaders often viewed the priorities differently. For example, system leaders saw alignment with the aim to improve self-management support for their patients as an overall goal, while clinicians at the practice were concerned with practical considerations of how and who would execute specific tasks related self-management support. We had to address both with messaging and training for all roles. Furthermore, we could not simply "drop" the intervention into the enrolled practices. All practices received basic training and education on SMS. Yet, once in the door, these early practice visits quickly showed that our recruitment effort was not finished.

There were 2 primary lessons from our experience: 1) More complex study interventions required more recruiting efforts with more stakeholders at more levels, at the clinic, in the system, and across roles; and 2) Recruitment is a process, not an event, and may take longer than expected with unplanned turnover of key contacts. The lessons and the strategies to improve recruiting appear in Table 1.

Of course, not all practices or systems we invited to participate chose to participate: some were simply not interested, others were interested but timing was not right because there was too much else going on, and several perceived the study as too much effort compared with potential benefits. One practice specifically noted the lack of sufficient financial incentives for participation; another did not want to be randomized to a nonintervention group.

\section{Discussion}

PBRN-led studies that require multi-layered changes in clinical workflow or staffing infrastructure require a tailored and strategic approach to practice recruitment. This strategy includes special attention to the length of the recruitment process, identifying and engaging multiple stakeholders at each clinical site around the project, and building a robust communication infrastructure to maintain engagement across the timeline of the study. System and practice leaders may open the door to their practices, but successful practice recruitment for practice-level pragmatic trials of more complex practice transformation interventions may require 


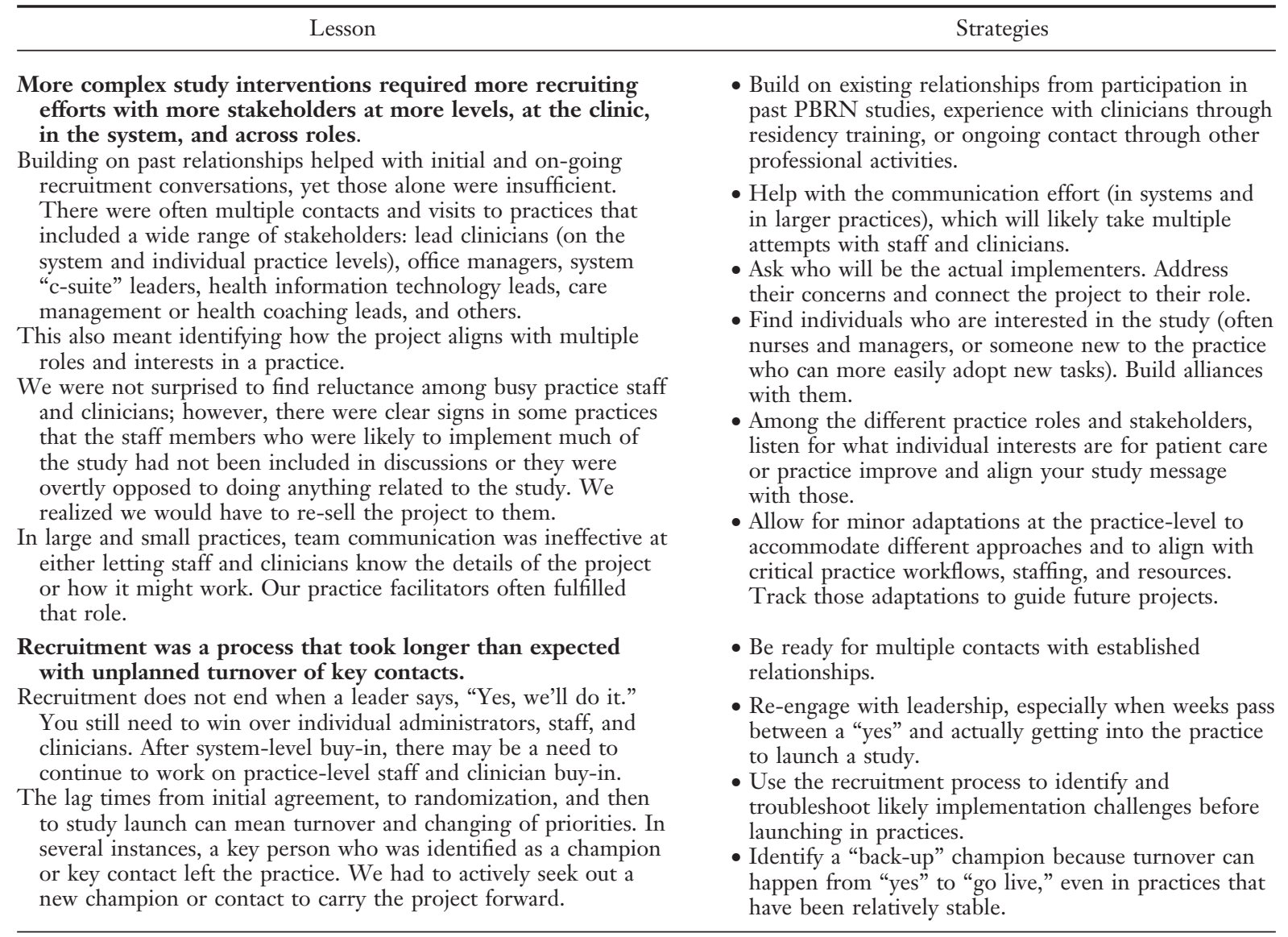

thoughtful multi-level personal outreach to identify "local" practice-level support throughout the practice-beyond the usual leadership-for sufficient engagement before starting an intervention. Recruiting practices (not just individual clinicians or staff) involves an ongoing process that cannot be neglected.

Recommendations that studies be clinically relevant, extend collaborations, provide health information technology help, make personal contact, and provide training are still germane. ${ }^{2,19}$ And, as others have learned, it works well to "make friends with the office personnel." 1 They serve as "gatekeeper" to the clinicians in the practice and are often the study implementers.

Our experience also reminds us that primary care practices differ in their strengths, which may not always be well aligned with the requirements of practice-based research studies. Capacities and capabilities can also change quickly, which was challenging because weeks or months separated formal recruitment and study launch at some practice sites. Further, some practices may lack the fundamental building blocks of a high-performing practice ${ }^{20}$ needed to effectively integrate research activities or necessary practice changes into their routine care workflows.

\section{Limitations}

The results reflect lessons learned from a single study focused on self-management support for patients with T2DM. Although notes were kept during the recruitment process, there were not detailed data (eg, interviews or field notes) on practices that did not enroll, so there may be other recruitment barriers and strategies we did not observe.

\section{Conclusion}

Study designs with a large number of practices may be challenging when recruiting over months or years. When undertaking a pragmatic clinical trial in a PBRN setting, it is important that PBRN leaders develop a comprehensive strategy to identify and engage a broad group of stakeholders within each practice, understand their needs and 
priorities around research, and design and implement a structured communications strategy to maintain engagement throughout every phase of the project.

To see this article online, please go to: bttp://jabfm.org/content/ 31/6/947.full.

\section{References}

1. Fulda KG, Hahn KA, Young RA, et al. Recruiting Practice-based Research Network (PBRN) physicians to be research participants: Lessons learned from the North Texas (NorTex) Needs Assessment Study. J Am Board Fam Med 2011;24:610-5.

2. Graham DG, Spano MS, Stewart TV, Staton EW, Meers A, Pace WD. Strategies for planning and launching PBRN research studies: A project of the Academy of Family Physicians National Research Network (AAFP NRN). J Am Board Fam Med 2007; 20:220-8.

3. Goodyear-Smith F, York D, Petousis-Harris H, et al. Recruitment of practices in primary care research: The long and the short of it. Fam Pract 2009;26: 128-36.

4. Johnston S, Liddy C, Hogg W, Donskov M, Russell G, Gyorfi-Dyke E. Barriers and facilitators to recruitment of physicians and practices for primary care health services research at one centre. BMC Med Res Methodol 2010;10:109.

5. Leathem CS, Cupples ME, Byrne MC, et al. Identifying strategies to maximise recruitment and retention of practices and patients in a multicentre randomised controlled trial of an intervention to optimise secondary prevention for coronary heart disease in primary care. BMC Med Res Methodol 2009;9:40.

6. Reed RL, Barton CA, Isherwood LM, Baxter JM, Roeger L. Recruitment for a clinical trial of chronic disease self-management for older adults with multimorbidity: A successful approach within general practice. BMC Fam Pract 2013;14:125.

7. Ngune I, Jiwa M, Dadich A, Lotriet J, Sriram D. Effective recruitment strategies in primary care research: A systematic review. Qual Prim Care 2012; 20:115-23.

8. Hudson SV, Harris-Haywood S, Stange KC, Orzano AJ, Crabtree BF. Recruiting minority primary care practices into practice-based research. Med Care 2006;44:696-700.

9. National Committee for Quality Assurance (NCQA). NCQA Patient-Centered Medical Home $(\mathrm{PCMH})$ Standards and Guidelines. 2017 Edition, Version 2 (Effective September 30, 2017). Washington, DC: National Committee for Quality Assurance (NCQA); 2017.

10. Centers for Medicare and Medicaid Services. CAHPS for MIPS Survey. Baltimore, MD; 2017.

11. Battersby M, Von Korff M, Schaefer J, et al. Twelve evidence-based principles for implementing selfmanagement support in primary care. Jt Comm J Qual Patient Saf 2010;36:561-70.

12. Glasgow RE, Dickinson P, Fisher L, et al. Use of RE-AIM to develop a multi-media facilitation tool for the patient-centered medical home. Implement Sci 2011;6:118.

13. Glasgow RE, McKay HG, Piette JD, Reynolds KD. The RE-AIM framework for evaluating interventions: What can it tell us about approaches to chronic illness management? Patient Educ Couns 2001;44:119-27.

14. Dickinson WP, Dickinson LM, Jortberg BT, Hessler DM, Fernald DH, Fisher L. A cluster randomized trial comparing strategies for translating selfmanagement support into primary care practices. BMC Fam Pract 2018;19:126.

15. Crabtree BF, Miller WL. Doing qualitative research. 2nd ed. Thousand Oaks, CA: Sage Publications; 1999.

16. Reis S, Biderman A, Mitki R, Borkan JM. Secrets in primary care: A qualitative exploration and conceptual model. J Gen Intern Med 2007;22:1246-53.

17. Crabtree BF, Miller WL, Crabtree BF, Miller WL. Using codes and code manuals. Vol 2. Thousand Oaks, CA: SAGE Publications;1999;163-77.

18. Miles MB, Huberman AM, Saldaña J. Qualitative data analysis: A methods sourcebook. 3rd ed. Thousand Oaks, CA: SAGE Publications; 2014.

19. Calmbach WL, Ryan JG, Baldwin LM, Knox L. Practice-based research networks (PBRNs): Meeting the challenges of the future. J Am Board Fam Med 2012;25:572-6.

20. Bodenheimer T, Ghorob A, Willard-Grace R, Grumbach K. The 10 building blocks of highperforming primary care. Ann Fam Med 2014;12: $166-71$. 